\title{
50 years on ... the discovery of tubulin continues to advance cancer treatment
}

\author{
Karen Crasta1,2,3,4 and Ritu Aneja5 \\ 'Lee Kong Chian School of Medicine, Nanyang Technological University, Singapore, Singapore \\ 2School of Biological Sciences, Nanyang Technological University, Singapore, Singapore \\ ${ }^{3} A *$ STAR Institute of Molecular and Cell Biology, Singapore, Singapore \\ ${ }^{4}$ Department of Medicine, Imperial College London, London, United Kingdom \\ ${ }^{5}$ Department of Biology, Georgia State University, Atlanta, Georgia, USA
}

Correspondence should be addressed to K Crasta or R Aneja Email kccrasta@ntu.edu.sg or raneja@gsu.edu
The existence of microtubules was known as early as the late 1800s, as documented by Boveri in elegant renderings of his observations of dividing cells (Satzinger 2008). It was not until the late 1940s, however, that the tubular ultrastructure of microtubules became evident due to improvements in fixation methods and electron microscopy. Based on their studies of nerve cells, De Robertis and Schmitt described them as 'neurotubules' (De Robertis 1968) betraying that the ubiquity of these structures across cell types was yet to be appreciated. This fact came to light in the early 1960s thanks to Slautterback's studies of hydra (Slautterback 1963) and Ledbetter and Porter's studies of plants (Wells 2006); these astute researchers then conferred on these tiny slender tubes their ultimate moniker (Wells 2006, Borisy et al. 2016). Shortly thereafter, the monumental achievement of discerning the fundamental component of the microtubule was made by Gary Borisy, at the time, a graduate student in the lab of Edwin Taylor at the University of Chicago. Borisy was interested in understanding the molecular mechanisms of cell division, and to this end, he sought to identify the protein that binds to colchicine and thereby antagonizes mitosis (Borisy et al. 2016). Thus, unfolded a bold journey to isolate tubulin, although he did not call it as such (Wells 2006, Borisy et al. 2016) (rather, Mohri, in his characterization of the amino acid composition of tubulin in sperm flagellae gave tubulin its quite fitting final appellation (Mohri 1968)). Borisy leveraged the high affinity of colchicine for the mitotic spindle to isolate, purify and characterize tubulin in 1967 (Borisy \& Taylor
1967). Since the colchicine-tubulin complex gave the same elution volume in gel-filtration and sedimentation coefficient in zone centrifugation in sea urchin eggs, cancer cells, spermatozoa and the brain, it was concluded that the same macromolecule was responsible for binding in all these sources. The increased binding activity detected for extracts of the mitotic spindle compared with whole sea urchin eggs further supported that the subunit of microtubules had indeed been isolated. Sea urchin eggs were an inconvenient source of tubulin; but Borisy soon found that tubulin was abundant in brain, a tissue that then fuelled the burgeoning field of microtubule research and facilitated efforts in subsequent decades to identify the molecular mechanisms of microtubule dynamic instability and treadmilling, microtubule-associated proteins, the various $\alpha-, \beta-, \gamma-, \delta-, \varepsilon-$, and $\zeta$-tubulin genes and a diversity of post-translational tubulin modifications, all of which presently inform the rational design of novel anti-tubulin drugs so often included in chemotherapy backbones (Borisy et al. 2016). For that reason, now half a century since that groundbreaking discovery, this special issue will appraise selected key topics, which deftly explore the consequences of microtubule dynamics for cancer progression and therapy. Topics and authors were carefully selected to provide the reader with cutting-edge information reflecting advances in the field.

Microtubule dynamics are critical for fundamental processes during cell division such as chromosome segregation, cytokinesis and general intracellular communication. Indeed, the basis of the cytotoxic action 
of microtubule-targeting drugs is a consequence of the perturbation of microtubule dynamics. We therefore begin with a perspective by Battaje \& Panda (2017) on lessons learnt from the bacterial homolog of tubulin Ftsz for microtubule dynamics. Here they examine evolutionary aspects and structural and functional homology. These provide enhanced understanding of the fundamental study of microtubule dynamics and offer potential implications for the design of novel chemotherapeutics.

Deviant kinetochore microtubule dynamics underlie chromosomal instability (CIN), defined as the elevated rate of gain or loss of whole or segmental chromosomes. Hence, CIN is a major cause of aneuploidy and is a common occurrence in many solid tumours. Two reviews in this special issue deal with the role of CIN in tumourigenesis: Sarah McClelland offers mechanistic insights into drivers of CIN in cancer progression and how CIN drives tumour evolution (McClelland 2017), whereas Chan \& Ngeow (2017) approach the same issues for CIN in the context of germline mutations in cancer predisposition syndromes. From these, a clear take-home message is that CIN represents a cancerspecific therapeutic target, as will be discussed later in this issue. A well-established cause of CIN, that had been suggested by Theodor Boveri more than a century ago, is centrosome amplification. Early studies of invasive breast tumours revealed that a majority of them are aneuploid and possess extra centrosomes. In this regard, Ritu Aneja and colleagues discuss the relationship of centrosome amplification with centrosome-clustering-mediated CIN and p53 mutation/loss in human breast tumours (Ogden et al. 2017). Notably, they provide breakthrough information about a new link between centrosome amplification, p53 mutations and racial health disparities in breast cancer outcome. These may provide riskstratification strategies for treatment regimens.

Finally, four comprehensive reviews that conclude this special issue provide exquisite clinically-relevant discussions centred around anti-mitotic therapeutic strategies. Agarwal \& Varma (2017) systematically review pathways targeting mitotic regulators with an emphasis on endocrine-related cancers. Shi \& Mitchison (2017) brilliantly discuss variation in cell-death response to drug treatment in cell culture, mouse tumour model and in patients. A key cell fate that accounts for this variation is the cellular process of mitotic slippage, which the review by Cheng \& Crasta (2017) next focuses on. The authors provide insights into the consequences of mitotic slippage, with potential implications for inflammation and drug resistance and represents a good segue into the final review by Visconti \& Grieco (2017). Here, the authors offer a thorough discussion on combating the two main limitations of anti-microtubule cancer drugs, namely drug resistance and toxicity with emphasis on prostate cancers and triple-negative breast cancers.

All the reviews covered here bring us to a pertinent question that the field is currently attempting to address: 'How do we predict which anti-microtubule drugs work and which don't in a patient?' As we enter into the era of personalized medicine, we hope that the information gleaned from this special issue will help you appreciate the significance of the landmark discovery of the tubulin and how research on microtubules continue to drive technological advances. Happy reading!

It was our utmost pleasure and honour working with all the experts who contributed to this special issue, both authors and reviewers of submitted manuscripts. We would also like to express our deepest gratitude to Prof Charis Eng (Editor-in-Chief) for giving us the opportunity as Guest Editors, as well as Alison Pope and Lucy Davidson, for all assistance rendered throughout this endeavour.

\section{References}

Agarwal S \& Varma D 2017 Targeting mitotic pathways for endocrinerelated cancer therapeutics. Endocrine-Related Cancer 24 T65-T82. (doi:10.1530/ERC-17-0080)

Battaje RR \& Panda D 2017 Lessons from bacterial homolog of tubulin, FtsZ for microtubule dynamics. Endocrine-Related Cancer 24 T1-T21. (doi:10.1530/ERC-17-0118)

Borisy GG \& Taylor EW 1967 The mechanism of action of colchicine. Colchicine binding to sea urchin eggs and the mitotic apparatus. Journal of Cell Biology 34 535-548.

Borisy G, Heald R, Howard J, Janke C, Musacchio A \& Nogales E 2016 Microtubules: 50 years on from the discovery of tubulin. Nature Reviews Molecular Cell Biology 17 322-328.

Chan SH \& Ngeow J 2017 Germline mutation contribution to chromosomal instability. Endocrine-Related Cancer 24 T33-T46. (doi:10.1530/ERC-17-0062)

Cheng B \& Crasta K 2017 Consequences of mitotic slippage for antimicrotubule drug therapy. Endocrine-Related Cancer 24 T97-T106. (doi:10.1530/ERC-17-0147)

De Robertis E 1968 The Miguel F. Jimenez lecture. Structural biochemical bases involving the transmission of nerve impulses. Gaceta Médica de México 98 689-703.

McClelland S 2017 Role of chromosomal instability in cancer progression. Endocrine-Related Cancer 24 T23-T31. (doi:10.1530/ ERC-17-0187)

Mohri H 1968 Amino-acid composition of 'Tubulin' constituting microtubules of sperm flagella. Nature 217 1053-1054.

Ogden A, Rida P \& Aneja R 2017 Centrosome amplification: a suspect in breast cancer and racial disparities. Endocrine-Related Cancer 24 T47-T64. (doi:10.1530/ERC-17-0072)
(C) 2017 Society for Endocrinology Printed in Great Britain
Published by Bioscientifica Ltd 
Satzinger H 2008 Theodor and marcella boveri: chromosomes and cytoplasm in heredity and development. Nature Reviews Genetics 9 231-238.

Shi J \& Mitchison T 2017 Cell death response to anti-mitotic drug treatment in cell culture, mouse tumor model and the clinic. Endocrine-Related Cancer 24 T83-T96. (doi:10.1530/ERC-17-0003)
Slautterback DB 1963 Cytoplasmic microtubules. I. Hydra. Journal of Cell Biology 18 367-388.

Visconti R \& Grieco D 2017 Fighting tubulin-targeting anticancer drug toxicity and resistance. Endocrine-Related Cancer 24 T107-T117. (doi:10.1530/ERC-17-0120)

Wells WA 2006 Microtubules get dynamic. Journal of Cell Biology 172643.

Received in final form 12 July 2017

Accepted 14 July 2017 